\title{
Some Recent Results on the 3C-SiC Structural Defects
}

\author{
A. Mantzari, A. Andreadou, M. Marinova* and E.K. Polychroniadis \\ Department of Physics, Aristotle University of Thessaloniki, 54124, Thessaloniki, Greece
}

\begin{abstract}
This work presents some recent results on the $3 \mathrm{C}-\mathrm{SiC}$ structural defects, studied by transmission electron microscopy. The samples were grown in several laboratories, using different methods. There has always been special attention to the region close to the interface between the seed and the overgrown material. This is due to the fact that this region is very important to the evolution of defects during growth. The main defects in SiC are micropipes, double position boundaries, stacking faults and dislocations. The defects that are most frequently observed in $3 \mathrm{C}-\mathrm{SiC}$ and more difficult to eliminate are inclusions of other polytypes, twins and microtwins and mainly stacking faults.
\end{abstract}

PACS: 68.37.Lp, 68.37.Og, 61.72.Nn, 61.72.Mm

\section{Introduction}

There are two main uses of silicon carbide ( $\mathrm{SiC})$. The first and oldest is as coating on ferrous substrates for the production of better cutting tools. The second is as substrate for the production of high temperature, and/or high power electronic devices. This is due to some characteristic properties of the material like the wide band gap [1], the high thermal conductivity (between 3 and $\left.5 \mathrm{~W} \mathrm{~cm}^{-1} \mathrm{C}^{-1}[2]\right)$, the high breakdown electric field and high saturated electron drift velocity. The combination of these properties makes $\mathrm{SiC}$ the best candidate for replacing $\mathrm{Si}$ in the microelectronic industry when a device has to work in harsh environment.

Among all polytypes, only one, $3 \mathrm{C}-\mathrm{SiC}$, has the cubic zinc-blende structure and the lowest band gap energy. 3C-SiC keeps a specific interest due to its highest electron mobility $\left(1000 \mathrm{~cm}^{2} \mathrm{~V}^{-1} \mathrm{~s}^{-1}[2,3]\right)$, highest electron saturation velocity $\left(2.5 \times 10^{7} \mathrm{~cm} \mathrm{~s}^{-1}[2,3]\right)$ and lower density of traps at the $3 \mathrm{C}-\mathrm{SiC} / \mathrm{SiO}_{2}$ interface. However, up to now growth of the material in a quality reasonable for electronic applications, i.e. with low defect density, is not possible. The purpose of this work is to present the main defects that appear in $3 \mathrm{C}-\mathrm{SiC}$ grown by different methods like chemical vapor deposition (CVD), vapor-liquid-solid mechanism (VLS), and liquid phase epitaxy (LPE) and their study by transmission electron microscopy (TEM).

\section{Results and discussion \\ 2.1. Structural characteristics}

$\mathrm{SiC}$ is distinguished by a specific structural characteristic which is polytypism i.e. a one-dimensional polymorphism. Si and $\mathrm{C}$ atoms are lying on separated close-packed planes. Two such planes (one Si plane and one $\mathrm{C}$ plane) form a $\mathrm{Si}-\mathrm{C}$ bilayer lying on a $\{111\}$ crystallographic orientation. All these bilayers are stacked

* Present address: CEA, LITEN, Nano-Characterisation Platform at Minatec, 17 rue des Martyrs, 38054 Grenoble Cedex 9, France. one over the other building the material. By this way, different stacking sequences give rise to different unit cells and accordingly to different polytypes. The ... ABCABC... sequence leads to a pure cubic structure $(3 \mathrm{C}-\mathrm{SiC})$ and the ...ABABAB... sequence leads to a pure hexagonal one $(2 \mathrm{H}-\mathrm{SiC})$. There are a lot of different polytypes, which are more than 200 according to international literature. Except for $2 \mathrm{H}-\mathrm{SiC}$ and 3C-SiC, already mentioned, the most common ones are: $4 \mathrm{H}-\mathrm{SiC}$ having the sequence ... ABCB. . , 6H-SiC having the sequence ... ABCACB... and 15R-SiC having the sequence ... ABCACBCABACABCB.... This specific structural property is the major reason for the defect peculiarities of $\mathrm{SiC}$.

\subsubsection{Stacking faults}

\subsection{Defects study}

Stacking faults (SFs) are the most frequently observed defects as well as the most difficult to avoid due to their very low energy of formation [4-6]. The very low energy necessary to locally change the stacking sequence in $\mathrm{SiC}$ polytypes results in the enormous structural variety of them. For example, the SF formation energies in $4 \mathrm{H}-$ and $6 \mathrm{H}-\mathrm{SiC}$ are measured to be 15 and $3 \mathrm{~mJ} / \mathrm{m}^{2}$, respectively, which is significantly lower compared to the corresponding SF formation energies of $\mathrm{Si}\left(55 \mathrm{~mJ} / \mathrm{m}^{2}\right)$ and GaAs $\left(45 \mathrm{~mJ} / \mathrm{m}^{2}\right)$ [7, 8]. Additionally, theoretical calculations show that for $3 \mathrm{C}-\mathrm{SiC}$ the $\mathrm{SF}$ energy has negative values namely about $-3.0 \mathrm{~mJ} / \mathrm{m}^{2}$. SFs always lie on $\{111\}$ planes and for this reason one has two possibilities to observe them and calculate their density, according to the crystallographic orientation of the samples studied in TEM. Figure 1a and b shows plane view (PV) images of $3 \mathrm{C}-\mathrm{SiC}$ in two different orientations. Figure 1a has a (111) orientation and thus only three of the four equivalent $\{111\}$ planes are observed, while in Fig. 1b, having an (001) orientation all four $\{111\}$ planes are present.

\subsubsection{Microtwins}

Twinning is another kind of defect that is usually observed. Growing 3C-SiC on a close-packed plane (i.e. heteroepitaxially on (0001) $\alpha$-SiC substrates), the first cubic 


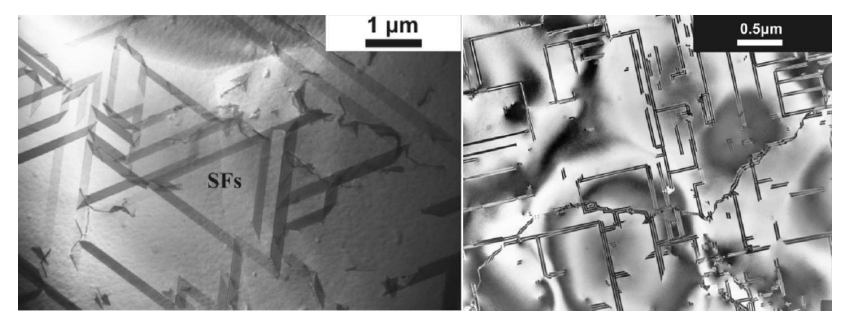

Fig. 1. PVTEM micrographs of 3C-SiC having (a) (111) orientation and (b) (001) orientation.

layer could start in two different orientations. Each one results from the other by a $60^{\circ}$ rotation along [111] which is the growth direction. If two islands in these two different orientations coalescence, immediately a twin boundary (TB) will be formed. An isolated twin boundary can be formed during crystal growth at high temperature. At lower temperatures after growth twins can be formed by motion of partial dislocations in adjacent "basal" planes [9]. For reasons that are not proved, during VLS process [10] one of these two orientations is energetically favorable and has a higher growth rate, covering the neighboring island and leaving only microtwins (MT) at the substrate/layer interface (Fig. 2). By this way, a mechanism of twin elimination is created.

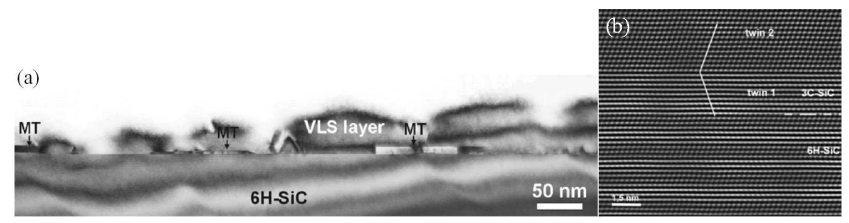

Fig. 2. (a) Cross-section image of 3C-SiC layer where $\mathrm{MTs}$, confined at the $3 \mathrm{C} / 6 \mathrm{H}-\mathrm{SiC}$ interface, are formed and (b) a high resolution image of a few bilayers thick such MT.

\subsubsection{Inversion domain boundaries}

This is a common defect when a polar material is grown on a non-polar substrate and in the case studied, when $\mathrm{SiC}$ is grown on $\mathrm{Si}$. Figure 3 shows such a case where the substrate used is (001) Si. In Fig. 3a there is a step on the substrate with a height of $a_{\mathrm{Si}} / 4$ along [001] and in Fig. 3b two adjacent grains are grown, one having on its first layer $\mathrm{Si}$ atoms and the other $\mathrm{C}$ atoms. In both cases a (110) inversion domain boundary (IDB) is formed.

Figure 4a shows two (110) IDBs which are propagating almost parallel to the [001] growth direction. As it is seen, the two boundaries progressively bend causing their mutual annihilation. The high resolution image of Fig. 4b shows a perfect (110) IDB which in another region starts to turn resulting in a zig-zag propagation. The high magnification image of Fig. 4c reveals that the IDB alternatively lies on $\{111\}$ planes. By this way the IDBs firstly lower their energy and secondly two by two annihilate themselves.

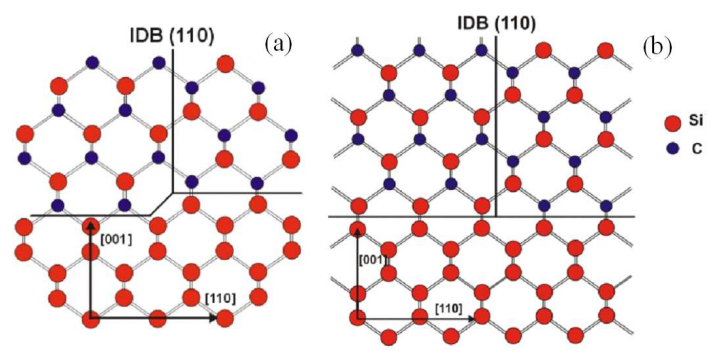

Fig. 3. Geometrical consideration for formation of an (110) IDB when (a) there is a step at the surface of the substrate with a height of $a_{\mathrm{Si}} / 4$ [001] and (b) two nuclei are in contact, one of which has $\mathrm{Si}$ atoms in the first layer and the other $\mathrm{C}$ atoms.

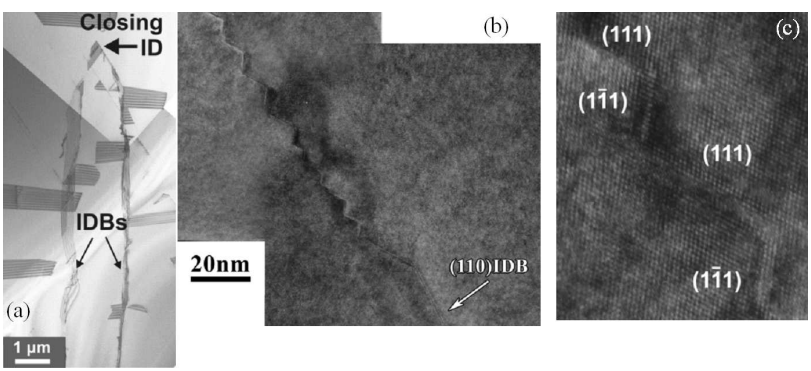

Fig. 4. (a) Cross-section micrographs where one large inversion domain is emanating, bounded by two IDBs, which are almost parallel to the growth direction. As the film grows, the IDBs progressively bend and finally close the inversion boundary. (b) High resolution (HR) TEM micrograph where it is obvious that the initial (110) IDB starts to turn resulting in a zig-zag propagation and (c) high magnification image of a thinner area of the specimen where it is seen that the IDB alternatively lies on $\{111\}$ planes.

\subsubsection{Inclusions of other polytypes}

The last type of frequently observed defects in the grown $3 \mathrm{C}-\mathrm{SiC}$ is the inclusions of other polytypes. Despite extended research, the driving forces of polytypism and instability of different polytypes are not yet clearly understood.

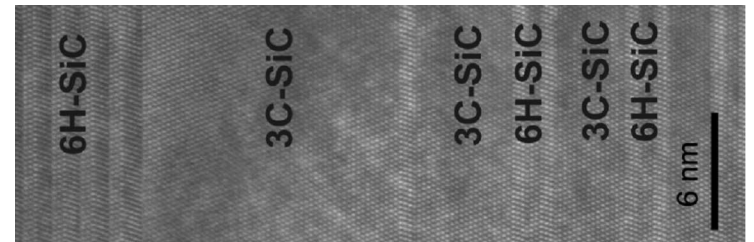

Fig. 5. HRTEM micrograph showing the mutual alternation of the $6 \mathrm{H}-\mathrm{SiC}$ and $3 \mathrm{C}-\mathrm{SiC}$ polytypes in a VLS layer.

Polytypic transformations usually take place either during the growth process or later during thermal processing. The most often observed are the $6 \mathrm{H}-\mathrm{SiC}$ ones which usually appear as a transition layer in the first 


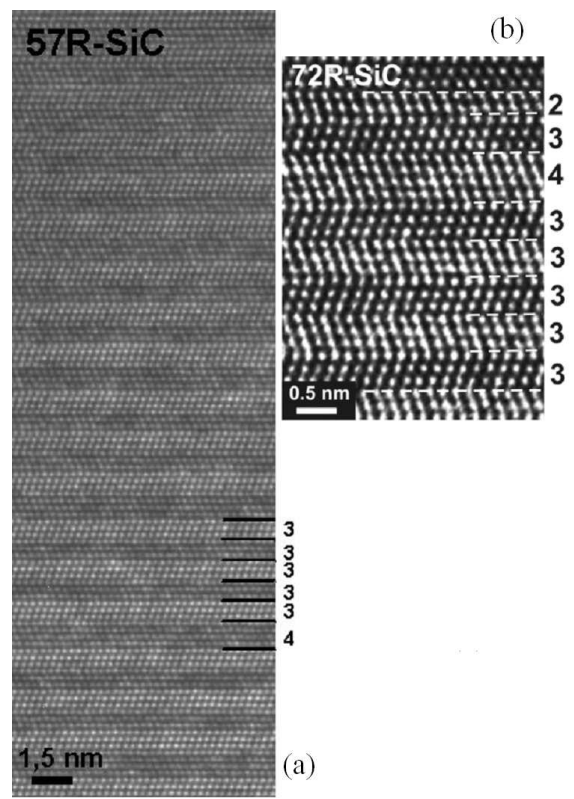

Fig. 6. HRTEM micrographs of (a) 57R-SiC and (b) $72 \mathrm{R}-\mathrm{SiC}$

stages of 3C-SiC grown on $6 \mathrm{H}-\mathrm{SiC}$ substrates (Fig. 5). Besides these, inclusions of other, in some cases long period, polytypes appear (like $57 \mathrm{R}-\mathrm{SiC}$ and $72 \mathrm{R}-\mathrm{SiC}$ shown in Fig. 6).

\section{Conclusions}

Conclusively, it was observed that the most stable defects that always appear and most difficult to be eliminated are the stacking faults. By controlling carefully the growth conditions a substantial reduction of their density can be achieved but it is almost up to now impossible to reach the upper limit of $10^{3} \mathrm{~cm}^{-1}$, which is necessary for any device fabrication.

\section{Acknowledgments}

The work presented has been performed within the MANSiC project (MRTN-CT-2006-035735) financially supported from the EU with FP6 and the SOLSIC European project (contract No. G5RD-CT-2001-00563). The authors are indebted to Dr. G. Ferro from UCBL 1 Lyon, Dr. D. Chaussende from INP-CNRS Grenoble and Dr. Adolf Schöner from ACREO AB for providing the samples.

\section{References}

[1] W.J. Choyke, D.R. Hamilton, L. Patrick, Phys. Rev. 144, A1163 (1964).

[2] Yu. Goldberg, M.E. Levinshtein, S.L. Rumyantsev, in: Properties of Advanced Semiconductor Materials GaN, AlN, SiC, BN, SiC, SiGe, Eds. M.E. Levinshtein, S.L. Rumyantsev, M.S. Shur, Wiley, New York 2001, p. 93.

[3] M. Bhatnagar, B.J. Baliga, IEEE Trans. Dev. 40, 645 (1993).

[4] H.P. Iwata, U. Lindelfelt, S. Öberg, P.R. Briddon, Phys. Rev. B 68, 245309 (2003).

[5] U. Lindefelt, H. Iwata, S. Öberg, P.R. Briddon, Phys. Rev. B 67, 155204 (2003).

[6] P. Käckell, Jürgen Furthmüller, F. Bechstedt, Phys. Rev. B 58, 1326 (1998).

[7] M.H. Hong, A.V. Samant, P. Pirouz, Philos. Mag. A 80, 919 (2000).

[8] M.H. Hong, A.V. Samant, P. Pirouz, Mater. Sci. Forum 338-342, 513 (2000).

[9] P. Pirouz, J.W. Yang, Ultramicroscopy 51, 189 (1993).

[10] G. Ferro, O. Kim-Hak, J. Lorenzzi, N. Jegenyes, M. Marinova, M. Soueidan, D. Carole, E.K. Polychroniadis, Mater. Sci. Forum 679-680, 71 (2011). 